\title{
TELSAM accelerates crystallization of fused target proteins by stabilizing weak crystal contacts

\author{
Supeshala Sarath Nawarathnage ${ }^{1}$, James Moody², Derick Bunn ${ }^{3}$, Nathan Towne ${ }^{4}$, Tzanko Duokov ${ }^{5}$ \\ ${ }^{1}$ No affiliation given ${ }^{2}$ No affiliation given, ${ }^{3}$ Brigham Young University, ${ }^{4}$ Brigham Young University, \\ ${ }^{5}$ Macromolecular Crystallography Group, Structural Molecular Biology Resource, SSRL
} supeshalawimalarathna@gmail.com
}

\begin{abstract}
Atomic-level structures of proteins are essential for structure-function studies and structure-based drug design. Xray crystallography is required for atomic-level structure determination of proteins too small for cryo-electron microscopy. Protein crystallization is the rate-limiting step in protein structure determination using X-ray diffraction. The Sterile Alpha motif (SAM) domain of human Translocation ETS Leukemia (TEL) protein is a chaperone that is engineered to polymerize at low $\mathrm{pH}$ (Kim et al. 2001). Investigation into covalent protein crystallization chaperones to facilitate the crystallization of novel protein targets is important as it increases the success rate of protein crystallization. The chaperone-target protein fusion polymerization lends avidity to strengthen subsequent weak crystal contacts made by the target protein. As the chaperone provides many of the crystal contacts, chaperones should enable protein crystallization with the minimal screening of crystallization conditions, significantly reducing the time and resources required to determine protein structure. The TELSAM monomers that polymerize when the $\mathrm{pH}$ is lowered are fused to target protein DARPin to test their ability to form diffraction-quality crystals. The 3TEL-DARPin structure showed that it forms a crystal lattice where polymers do not touch in 1 dimension and stabilize extremely weak inter-DARPin contacts between sheets of polymers. We then crystallized the human TNK1 UBA domain as a fusion to TELSAM. The UBA homology model that was used for the molecular replacement did not fit the electron density data. But the TELSAM polymer allowed the solution of the X-ray phases to build the UBA domain one helix at a time. With all these data we understand that the TELSAM helps to crystallize the target proteins 27 times faster. With the 1TEL-Flex-UBA structure, we learned that TELSAM polymers do not touch each other, and TELSAM crystallizes UBA with a lack of TELSAM polymers 1TEL-Rigid-UBA. In the future, $\mathrm{pH}$-dependent TELSAM can be used as a protein crystallization tool that speeds the crystallization of target proteins with diffraction quality crystals.
\end{abstract}

